\title{
Very Interesting Presentation: \\ VIP Co-secretion by a Phaeochromocytoma
}

\author{
CASE HISTORY 62 year old lady \\ Medical history and presentation: \\ 15 years ago - diagnosed with irritable bowel syndrome with alternating constipation and diarrhoea \\ 5 years ago - diarrhoea became the dominant feature, bowel opening every 20 minutes daily \\ Investigations negative for hyperthyroidism and coeliac disease but she was found to have a non-functioning anal sphincter \\ 3 years ago - a colostomy was performed privately to improve her symptoms, but large volumes of stool continued to be passed daily. She \\ had experienced episodes of palpitations and sweating, although these had resolved and appeared to coincide with menopause. \\ August 2011, right upper quadrant abdominal pain leading to admission under the Hepatico-pancreatico-biliary surgeons . CT imaging was \\ performed and subsequent referral made to Endocrinology. \section{as}

Other Past Medical History: Hysterectomy for menorrhagia 2001, Pernicious anaemia

Drug History: Amitriptyline, Omeprazole, B12

Examination: Normotensive, normal heart rate, no phenotype of Cushing's syndrome, no skin rashes

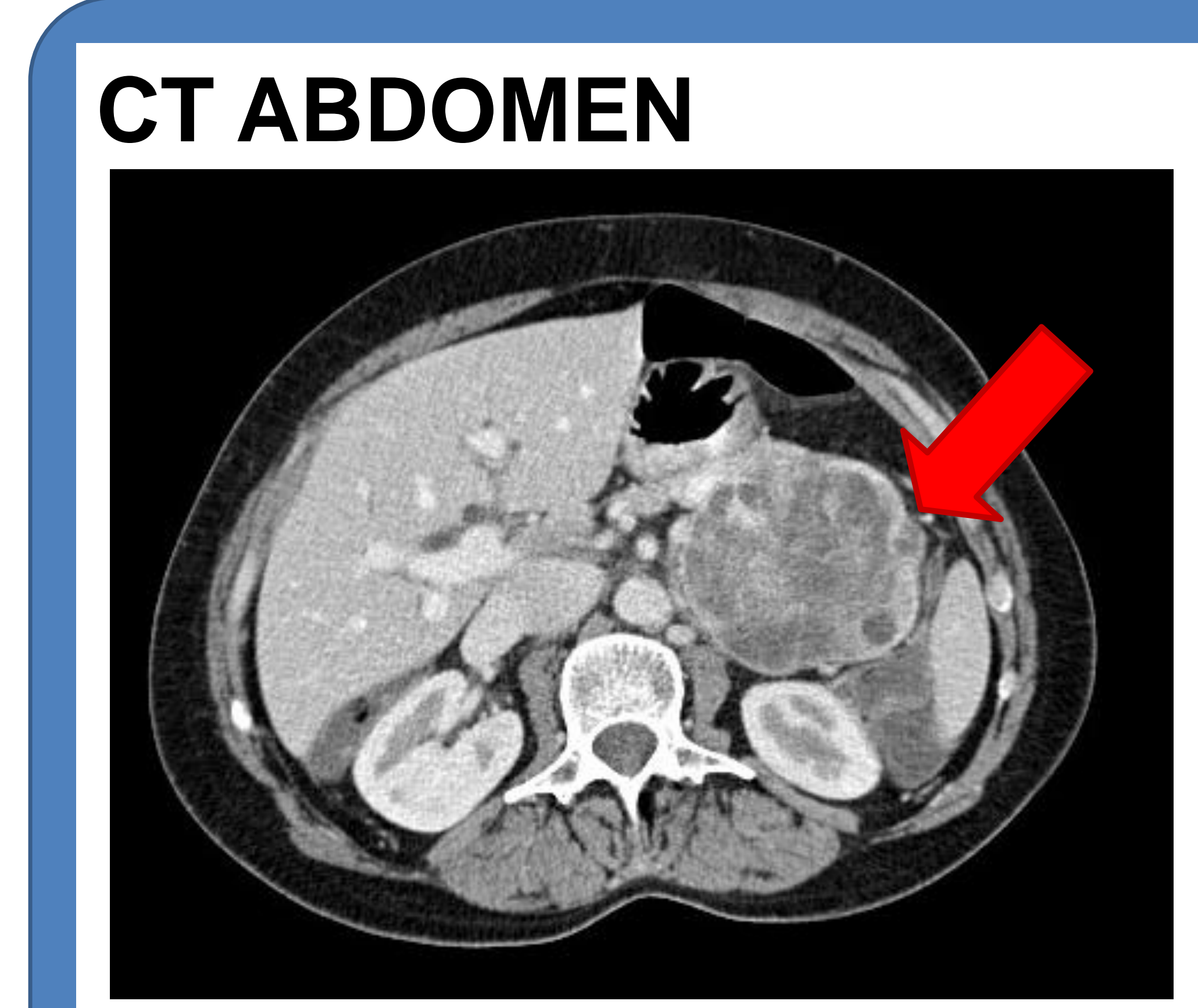

$7 \mathrm{~cm}$ multi-cystic tumour

Extending to the tail of the pancreas

Arising from the pancreas/left adrenal gland

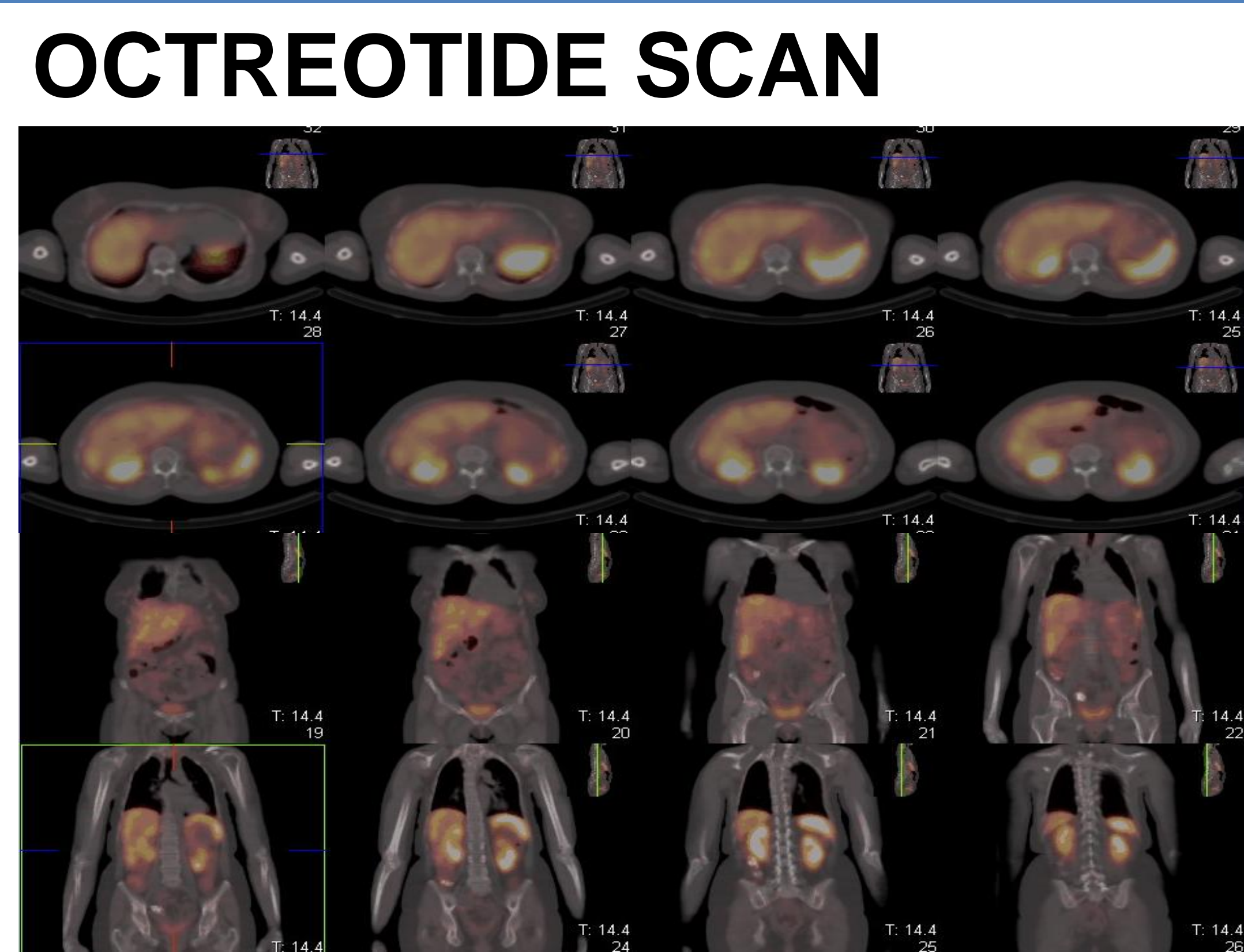

Mildly avid uptake on MIBG

\begin{tabular}{|c|c|c|c|c|c|c|c|}
\hline \multicolumn{8}{|c|}{$\begin{array}{l}\text { INVESTIGATIONS } \\
\text { Due to clinical suspicion plasma metanephrines were sent in addition to urinary catecholamines and demonstrated excess } \\
\text { noradrenaline secretion. }\end{array}$} \\
\hline \multicolumn{4}{|l|}{ CATECHOLAMINES } & \multicolumn{4}{|l|}{ GUT HORMONES } \\
\hline Urinary Adrenalline & 37 & nmol/24 Hrs & $<100$ & Vasoactive intestinal peptide & 100.0 & pmol/L & $<30.0$ \\
\hline Urinary Noradrenaline & 694 & nmol/24 Hrs & $<800$ & Pancreatic polypeptide & 43 & pmol/L & $<300$ \\
\hline Dopamine & 12768 & nmol/24 Hr & $<3100$ & Gastrin & 24 & pmol/I & $<40$ \\
\hline \multicolumn{4}{|l|}{ METANEPHRINES } & Glucagon & 10.0 & pmol/I & $<50.0$ \\
\hline Plasma Normetanephrine & 18562 & pmol/L & $120-1180$ & Somatostatin & 95 & pmol/L & $<150$ \\
\hline Plasma Metanephrine & 18328 & pmol/L & $80-510$ & Chromogranin A & 35 & pmol/L & $<60$ \\
\hline Urinary Normetadrenaline & 11.2 & Umol/24Hrs & & Chromogranin B & 94 & pmol/L & $<150$ \\
\hline Urinary Metadrenaline & 0.4 & Umol/24Hrs & & & & & \\
\hline
\end{tabular}

\section{SURGICAL RESECTION} PRE-OPERATIVE MANAGEMENT Appropriately alpha and beta blocked

Admitted 3 days pre-operatively to optimize blood pressure and circulating volume.

Proceeded to left adrenalectomy

No hypoglycaemia; Haemodynamically stable peri- and post-operatively

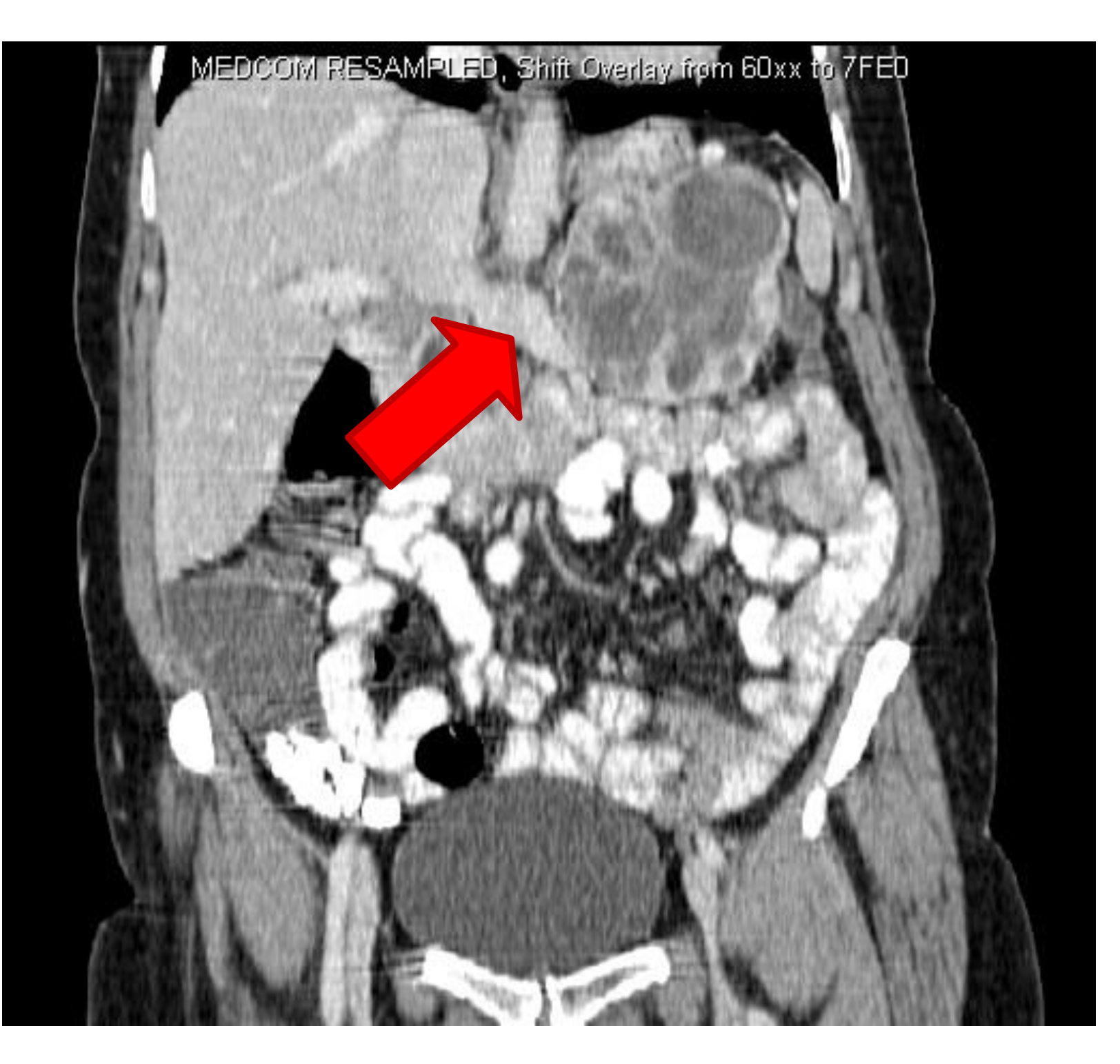

